(c) 2006 International Press

Adv. Theor. Math. Phys. 10 (2006) 283-315

\title{
Branes, moduli spaces and smooth transition from big crunch to big bang
}

\author{
Claus Gerhardt
}

Ruprecht-Karls-Universität, Institut für Angewandte Mathematik, Im Neuenheimer Feld 294, 69120 Heidelberg, Germany

gerhardt@math.uni-heidelberg.de

\begin{abstract}
We consider branes $N$ in a Schwarzschild- $\mathrm{AdS}_{(n+2)}$ bulk, where the stress-energy tensor is dominated by the energy density of a scalar fields map $\varphi: N \rightarrow \mathcal{S}$ with potential $V$, where $\mathcal{S}$ is a semi-Riemannian moduli space. By transforming the field equation appropriately, we get an equivalent field equation that is smooth across the singularity $r=0$, and which has smooth and uniquely determined solutions which exist across the singularity in an interval $(-\epsilon, \epsilon)$. Restricting a solution to $(-\epsilon, 0)$ resp. $(0, \epsilon)$, and assuming $n$ odd, we obtain branes $N$ resp. $\hat{N}$ which together form a smooth hypersurface. Thus a smooth transition from big crunch to big bang is possible both geometrically as well as physically.
\end{abstract}

e-print archive: http://lanl.arXiv.org/abs/hep-th/0409123 


\section{Introduction}

Finding a smooth transition from big crunch to big bang is an interesting and challenging problem in general relativity. For abstract spacetimes, i.e., for spacetimes that are not embedded in a bulk space, it is even a nontrivial question how to define a smooth transition. Using the inverse mean curvature flow in combination with a reflection we proved in $[2,3]$ that a smooth transition is possible for a certain class of spacetimes which we called Asymptotically Robertson-Walker (ARW) spaces.

For branes the phrasing of the problem is simpler and also the proof that there are solutions to the problem is much easier - at least, if the stressenergy tensor on the brane is made up of perfect fluids satisfying an equation of state with constant $\omega$, and of course, under the general assumption of radial symmetry, cf. [4].

If scalar fields with non-trivial potentials are considered in the stressenergy tensor, then the problem is much harder to solve, since the $\omega$ in the equation of state is not constant, and hence, the conservation law cannot be easily integrated.

We look at branes $N$ in a Schwarzschild-AdS ${ }_{(n+2)}$ bulk $\mathcal{N}=\mathbb{R}_{-} \times \mathbb{R} \times \mathcal{S}_{0}$, where $\mathcal{S}_{0}$ is an $n$-dimensional space form, assuming that the branes are located in the black hole region.

The relation between the geometry of the brane and physics is governed by the Israel junction condition.

Let $\Omega \subset \mathcal{N}$ be an open set contained in the black hole region, which is bounded by (part of) the horizon and two branes (timelike hypersurfaces) $N_{i}, i=1,2$, where $N_{2}$ may be empty.

The field equations are derived from an action principle, where the action integral $I$ is given by

$$
I=\int_{\Omega}(\tilde{R}+2 \Lambda)+\sum_{i=1}^{2} \int_{N_{i}}\left(2 H_{i}+4 \kappa\left(\sigma_{i}+{ }_{i} L_{\text {tot }}\right)\right) .
$$

$\tilde{R}$ is the scalar curvature of $\mathcal{N}, \Lambda$ a cosmology constant, $H_{i}$ the resp. mean curvature of the $N_{i}$, with respect to their inner normal, i.e., with respect to the normal that is pointing towards $\Omega, \sigma_{i}$ their tensions, and ${ }_{i} L_{\text {tot }}$ the resp. Lagrange functions corresponding to $N_{i}$; the Lagrange functions may be different, but are assumed to have the same structure, so that it suffices to described only the case $i=1$ in detail, where we omit the index 1 dealing 
instead with a generic brane $N . L_{\text {tot }}$ is then defined by

$$
L_{\mathrm{tot}}=\sum_{k=0}^{k_{0}} L_{k}+\left(-\frac{1}{2}\|D \varphi\|^{2}-V(\varphi)\right),
$$

where $L_{k}$ are Lagrange functions corresponding to perfect fluids satisfying an equation of state with constant $\omega_{k}$

$$
p_{k}=\frac{\omega_{k}}{n} \rho_{k}
$$

and $\varphi=\left(\varphi^{A}\right)$ is a map

$$
\varphi: N \longrightarrow \mathcal{S}
$$

where the moduli space $\mathcal{S}$ is an arbitrary semi-Riemannian manifold of finite dimension with metric $\left(g_{A B}\right)$ and $V$ a smooth potential. ${ }^{1}$ The Lagrange function

$$
L=-\frac{1}{2}\|D \varphi\|^{2}-V(\varphi)=-\frac{1}{2} g_{A B} \varphi_{\alpha}^{A} \varphi_{\beta}^{B} \bar{g}^{\alpha \beta}-V(\varphi)
$$

can be looked at as the energy density of the map $\varphi$.

The power law Lagrangians $L_{k}$ are only included for good measure, because they have already been treated in [4] and pose only a slight additional challenge as long as the $\omega_{k} \in \mathbb{Z}$ are bounded by

$$
\omega_{k} \leq n \text {. }
$$

The only interesting Lagrangian is the one given in (1.5).

In our previous paper, where we treated power law Lagrangians, we first solved the embedding problem in $\mathcal{N}$, then reflected the bulk space by switching the light cone and changing the radial coordinate from $r$ to $-r$ to obtain a new bulk $\hat{\mathcal{N}}$ which had a white hole singularity in $r=0$. The spaces $\mathcal{N}$ and $\hat{\mathcal{N}}$ could be pasted together to yield $\mathbb{R}^{2} \times \mathcal{S}_{0}$ topologically.

Then we used a reflection method to create a reflected brane in $\hat{N} \subset \hat{\mathcal{N}}$ such that $N \cup \hat{N}$ would form a smooth hypersurface in $\mathbb{R}^{2} \times \mathcal{S}_{0}$.

In the present case, where the stress-energy tensor corresponding to the scalar fields map will dominate the evolution of the brane near the singularity, a much better, if not optimal, result can be proved.

By choosing the radial coordinate as embedding parameter we are able to transform the field equation of the brane near the singularity to obtain a new equation which is smooth across the singularity and has smooth solutions which are uniquely determined by given initial values at the singularity.

\footnotetext{
${ }^{1}$ Note that $\mathcal{S}$ and $V$ can in general be different for different branes.
} 
This new field equation has to be satisfied near the singularity by any solution of the embedding problem provided its rescaled energy density $\rho r^{2 n}$ has a positive limit when the singularity is approached and the limit of the rescaled potential $V r^{2 n}$ vanishes.

If we assume that $n$ and the $\omega_{k}$ are odd, then we can show that the abstract solution of the new field equation is actually a physical solution on both sides of the singularity, and it exists in a small interval $\{-\epsilon<r<\epsilon\}$.

The action integrals $I$ in $\mathcal{N}$ resp. $\hat{\mathcal{N}}$ will be identical with the exception that, if we decompose a (globally) smooth brane in its parts $N \subset \mathcal{N}$ and $\hat{N} \subset \hat{\mathcal{N}}$ and if $\Omega$ resp. $\hat{\Omega}$ are the corresponding domains, then $N$ resp. $\hat{N}$ have opposite positions with respect to their domains, e.g., if $\Omega$ lies below $N$, in which case $N$ is called an upper brane, then $\hat{N}$ is a lower brane, i.e., $\hat{\Omega}$ lies above $\hat{N}$. Notice that the branes are graphs so that the $t$-axis provides a direction.

This behaviour is also responsible for the fact that a configuration with two boundary branes has a smooth "mirror" image in $\hat{\mathcal{N}}$, if and only if the branes collide at the singularity and switch positions in $\hat{\mathcal{N}}$.

It is remarkable that a smooth transition from big crunch to big bang is possible without additional symmetry assumptions on the potential or on the moduli space.

However, if $\mathcal{S}$ is locally symmetric and if there exists a point $p \in \mathcal{S}$ such that $V$ is invariant in a neighbourhood of $p$ with respect to the geodesic symmetry at $p$, then, by choosing $p$ as one initial value at the singularity, it can be shown that the solutions in $\mathcal{N}$ resp. $\hat{\mathcal{N}}$ correspond to each other via reflection near the singularity, after having introduced Riemannian normal coordinates at $p$.

Moreover, if $\mathcal{S}$ is Riemannian and complete and the energy density $\rho$ bounded from below, then we can prove that the branes extend to the resp. horizons, i.e., they are defined in $\left(-r_{0}, r_{0}\right)$, where $r_{0}$ is the black (white) hole radius.

At the horizon our embedding experiences a coordinate singularity. Thus, an extension past the horizon seems possible but we didn't pursue this point.

In order to describe our results accurately, let us introduce some definitions and notations.

The metric in the bulk space $\mathcal{N}$ is given by

$$
d \tilde{s}^{2}=-\tilde{h}^{-1} d r^{2}+\tilde{h} d t^{2}+r^{2} \sigma_{i j} d x^{i} d x^{j},
$$


where $\left(\sigma_{i j}\right)$ is the metric of an $n$-dimensional space form $\mathcal{S}_{0}$, the radial coordinate $r$ is assumed to be negative, $r<0$, and $\tilde{h}(r)$ is defined by

$$
\tilde{h}=m(-r)^{-(n-1)}+\frac{2}{n(n+1)} \Lambda r^{2}-\tilde{\kappa},
$$

where $m>0$ and $\Lambda \leq 0$ are constants, and $\tilde{\kappa}=-1,0,1$ is the curvature of $\mathcal{S}_{0}$. We note that we assume that there is a black hole region, i.e., if $\Lambda=0$, then we have to suppose $\tilde{\kappa}=1$.

We consider branes $N$ contained in the black hole region $\left\{-r_{0}<r<0\right\}$ given by an embedding

$$
y\left(\tau, x^{i}\right)=\left(r(\tau), t(\tau), x^{i}\right), \quad-a<\tau<0,
$$

with a big crunch singularity in $\tau=0$ such that $r(0)=0 . \quad N$ will be a globally hyperbolic spacetime $N=I \times \mathcal{S}_{0}$ with metric $\left(\bar{g}_{\alpha \beta}\right)$ that can be expressed in the so-called conformal time gauge as

$$
d \bar{s}^{2}=\mathrm{e}^{2 f}\left(-\left(d x^{0}\right)^{2}+\sigma_{i j} d x^{i} d x^{j}\right)
$$

such that

$$
f=f\left(x^{0}\right)=\log \left(-r\left(x^{0}\right)\right)
$$

Another gauge is even more important for our purposes, namely, by choosing $x^{0}=r$ as time function. The embedding then has the form

$$
y\left(r, x^{i}\right)=\left(r, t(r), x^{i}\right), \quad r<0,
$$

and the metric is given by

$$
d \bar{s}^{2}=-\left(\tilde{h}^{-1}-\tilde{h}\left|t^{\prime}\right|^{2}\right) d r^{2}+r^{2} \sigma_{i j} d x^{i} d x^{j} .
$$

The relation between geometry and physics is governed by the Israel junction conditions

$$
h_{\alpha \beta}-H \bar{g}_{\alpha \beta}=\kappa\left(T_{\alpha \beta}-\sigma \bar{g}_{\alpha \beta}\right) \text {, }
$$

where $h_{\alpha \beta}$ is the second fundamental form of $N, H=\bar{g}^{\alpha \beta} h_{\alpha \beta}$ the mean curvature, $\kappa \neq 0$ a constant, $T_{\alpha \beta}$ the stress-energy tensor and $\sigma$ the tension of the brane. 
Let $\omega_{k}, 0 \leq k \leq k_{0}$, be the constants in (1.3), where $\omega_{0}$ is assumed to satisfy

$$
\omega_{0}=n,
$$

and let $0 \leq \bar{\rho}_{k}$ be the corresponding integration constants. Define

$$
\mathcal{C}=\sum_{k=0}^{k_{0}} \bar{\rho}_{k}(-r)^{-\left(n+\omega_{k}\right)}, \quad \hat{\mathcal{C}}=(\sigma+\mathcal{C}) r^{2 n}, \quad \hat{h}=\tilde{h} r^{2 n}
$$

and

$$
D^{2}=1-2\left(\kappa^{2} / n^{2}\right)\|\dot{\varphi}\|^{2}(V+\sigma+\mathcal{C}) r^{2 n}-\left(\kappa^{2} / n^{2}\right) \tilde{h}\|\dot{\varphi}\|^{4}
$$

The main results of this paper can now be summarized in the following three theorems.

Theorem 1.1. Let $\mathcal{S}$ be semi-Riemannian with metric $\left(g_{A B}\right)$ and assume that the metric has at least one positive eigenvalue. The potential $V$ should be of class $C^{\infty}(\mathcal{S})$.

(i) Consider the action integral (1.1) for a single brane and look at embeddings of the form (1.12) parametrized by $r$ with a corresponding scalar fields map $\varphi(r)$. The field equations for $\varphi$ resulting from the Israel junction conditions and the zero divergence of the stress-energy tensor associated with $\varphi$, can be combined to a new field equation of the form

$$
\frac{D}{d r} \chi^{A}=F^{A}(r, \varphi, \chi)
$$

where

$$
\chi^{A}=\dot{\varphi} r^{-(n-1)}
$$

provided the term $D^{2}$ in (1.17) is strictly positive.

Treating the arguments of $F^{A}$ as independent variables $(r, \varphi, \chi)$, there holds $F^{A} \in C^{\infty}\left((-\epsilon, \epsilon) \times \mathcal{S} \times T^{1,0}(\mathcal{S})\right)$ as long as $\|\chi\|^{2}>0$ and $\epsilon$ is small, more precisely,

$$
\begin{aligned}
F^{A}= & -\frac{\hat{h}^{\prime}+2\left(\kappa^{2} / n^{2}\right)\left(\rho r^{2 n}+\hat{\mathcal{C}}\right)\left(2 n V r^{2 n-1}+\hat{\mathcal{C}}^{\prime}\right)}{\hat{h}+\left(\kappa^{2} / n^{2}\right)\left(\rho r^{2 n}+\hat{\mathcal{C}}\right)^{2}} \chi^{A} \\
& -\frac{\partial V}{\partial \varphi_{A}} \frac{r^{3 n-1}}{\hat{h}+\left(\kappa^{2} / n^{2}\right)\left(\rho r^{2 n}+\hat{\mathcal{C}}\right)^{2}}
\end{aligned}
$$

where the term $\rho r^{2 n}$ can also be expressed by $(r, \varphi, \chi)$. 
These field equations have to be satisfied near the singularity by any solution of the embedding problem that also solves the wave equation (3.10), provided the corresponding quantities $\rho r^{2 n}$ and $V r^{2 n}$ obey

$$
\lim _{r \rightarrow 0} \rho r^{2 n}>\bar{\rho}_{0}
$$

and

$$
\lim _{r \rightarrow 0} V r^{2 n}=0
$$

(ii) The field equations (1.18) can be solved abstractly in a full neighbourhood of the singularity $r=0$ with given initial values $\varphi(0), \chi(0)$, provided $\|\chi(0)\|^{2}>0$ and

$$
2\left(\kappa^{2} / n^{2}\right)\|\chi(0)\|^{2} \bar{\rho}_{0}<1
$$

for details we refer to Theorem 3.1. The solutions $(\varphi, \chi)$ are smooth in $(-\epsilon, \epsilon)$ and are related by

$$
\dot{\varphi}=\chi r^{n-1} .
$$

If we restrict $\varphi$ to $(-\epsilon, 0)$, then it is a solution of the original embedding problem in $\mathcal{N}$. The function $t(r)$ is smooth in $(-\epsilon, 0]$. This result holds for arbitrary $n$.

(iii) If in addition $n$ and the $\omega_{k}$ 's are odd, then the globally defined abstract solution in (i) also defines an embedded brane $\hat{N} \subset \hat{\mathcal{N}}$. If $N$ is an upper brane, then $\hat{N}$ is a lower brane and vice versa. The joint branes form a smooth hypersurface in $\mathbb{R}^{2} \times \mathcal{S}_{0}$ which is a graph $t=t(r), t \in C^{\infty}((-\epsilon, \epsilon))$.

(iv) If $\mathcal{S}$ is Riemannian and complete, and $\rho$ bounded from below, then the branes $N$ resp. $\hat{N}$ extend to the horizons. They probably extend past the horizons, but the embeddings experience a coordinate singularity at the horizons.

Theorem 1.2. If in addition to the assumptions in part (iii) of the preceding theorem, $\mathcal{S}$ is locally symmetric, and there exists $p \in \mathcal{S}$ such that the potential $V$ is invariant in a neighbourhood of $p$ with respect to the geodesic symmetry centered at $p$, then, if $p$ is chosen as initial value in (iii), the solution $\varphi$, after the introduction of Riemannian normal coordinates around $p$, is odd in a neighbourhood of $r=0$, and the brane $\hat{N}$ is the odd reflection of the brane $N$ near the singularity.

Theorem 1.3. Consider the action integral (1.3) with two boundary branes. If the assumptions of part (iii) of Theorem 1.1 are satisfied, then the configuration $\left(\Omega, N_{1}, N_{2}\right)$ has a smooth correspondence $\left(\hat{\Omega}, \hat{N}_{1}, \hat{N}_{2}\right)$ in $\hat{\mathcal{N}}$, only if the branes $N_{1}$ and $N_{2}$ collide at the singularity. They then switch positions in $\hat{\mathcal{N}}$. If the assumptions of part (iii) of Theorem 1.1 are valid, 
then this condition is also sufficient. Moreover, the requirement of colliding boundary branes at $r=0$ can be easily met.

\section{Domain walls}

The configuration in the previous section, with a domain $\Omega \subset \mathcal{N}$ bounded by one or two branes, can be generalized by assuming that a brane $N$ separates two subdomains $\Omega_{k} \subset \mathcal{N}_{k}, k=1,2$, where $\mathcal{N}_{k}$ are different bulk spaces, and where it is assumed that the induced metric of the brane from either side is the same.

In many papers it has been assumed that this configuration is possible for bulk spaces and embeddings where the ambient metrics have the form as in (1.7) and the embedding of the brane is given by (1.9), cf., e.g., [5]. We shall show that this is not possible. The only allowed difference between the bulk spaces in the described setting would be one caused by $\mathbb{Z}_{2}$ symmetry.

Proposition 2.1. Let $N$ be a brane separating two subdomains of Schwarzschild-AdS $S_{(n+2)}$ bulk spaces $\mathcal{N}_{k}, k=1,2$, with metrics given by

$$
d \tilde{s}_{k}^{2}=-\tilde{h}_{k}^{-1} d r^{2}+\tilde{h}_{k} d t^{2}+r^{2} \sigma_{i j} d x^{i} d x^{j},
$$

where $\left(\sigma_{i j}\right)$ is the metric of an $n$-dimensional space form $\mathcal{S}_{0}$, the radial coordinate $r$ can be either negative or positive, and is allowed to have different signs in the bulk spaces, and $\tilde{h}_{k}(r)$ is defined by

$$
\tilde{h}_{k}=m_{k}|r|^{-(n-1)}+\frac{2}{n(n+1)} \Lambda_{k} r^{2}-\tilde{\kappa},
$$

where $m_{k} \geq 0$ and $\Lambda_{k}$ are constants, and $\tilde{\kappa}=-1,0,1$ is the curvature of $\mathcal{S}_{0}$. The induced metric on the brane from both sides should be same. If we then assume that the embedding of $M$ is given by

$$
y(\tau)=\left( \pm r(\tau), \pm t(\tau), x^{i}\right), \quad r<0,
$$

where the plus sign corresponds to the case that the $r$ coordinate in $\mathcal{N}_{1}$ or in $\mathcal{N}_{2}$ is negative, then there must hold

$$
\tilde{h}_{1}=\tilde{h}_{2}
$$

i.e., the only possible difference between $\mathcal{N}_{1}$ and $\mathcal{N}_{2}$ is a $\mathbb{Z}_{2}$ symmetry.

Proof. We have written the metric in a form that suits our particular assumption that the branes are located in the black hole region. However, this is no restriction, since we do not assume any sign condition on $\tilde{h}_{k}$, i.e., $\tilde{h}_{k}$ could be negative and the bulk spaces could be AdS or dS spaces; in fact only the structure of the metric in (2.1) is relevant with arbitrary 
$\tilde{h}_{k}$ - satisfying $\tilde{h}_{k}(r)=\tilde{h}_{k}(-r)$, if we want to include $\mathbb{Z}_{2}$ symmetry, without any assumptions on the curvature.

The induced metric can be written as

$$
d \bar{s}^{2}=r^{2}\left(-\left(d x^{0}\right)^{2}+\sigma_{i j} d x^{i} d x^{j}\right) .
$$

We also assume that $\dot{r}>0$. Notice that $r<0$, so that $\left(x^{\alpha}\right)$ is a future oriented coordinate system on the brane.

Let us point out that this choice of $\tau$ implies the relation

$$
r^{2}=\tilde{h}_{k}^{-1} \dot{r}^{2}-\tilde{h}_{k}\left|t^{\prime}\right|^{2},
$$

since

$$
\bar{g}_{00}=\langle\dot{y}, \dot{y}\rangle,
$$

or equivalently,

$$
|\dot{r}|^{2}=\tilde{h}_{k} r^{2}+\tilde{h}_{k}^{2}\left|t^{\prime}\right|^{2}
$$

We assume furthermore that there is an open interval $I$ such that $y(\tau)$, for $\tau \in I$, separates corresponding regions in $\mathcal{N}_{k}$, i.e., $y(\tau)$ is either inside the event horizons - if they exist — or outside. Without loss of generality let us suppose that we are inside the black hole regions, so that $\tilde{h}_{k}>0$, $k=1,2$, otherwise we simply exchange the roles of $r$ and $t$, and write $h_{k}$ for $-\tilde{h}_{k}^{-1}$ and $-h_{k}^{-1}$ for $\tilde{h}_{k}$.

Subtracting equation (2.8) for $k=1$ resp. $k=2$, we get

$$
0=\left(\tilde{h}_{1}-\tilde{h}_{2}\right) r^{2}+\left(\tilde{h}_{1}^{2}-\tilde{h}_{2}^{2}\right)\left|t^{\prime}\right|^{2}
$$

and, thus, if $\tilde{h}_{1} \neq \tilde{h}_{2}$,

$$
0=r^{2}+\left(\tilde{h}_{1}+\tilde{h}_{2}\right)\left|t^{\prime}\right|^{2},
$$

a contradiction. Hence, the equality $\tilde{h}_{1}=\tilde{h}_{2}$ must be valid.

If the bulk spaces $\mathcal{N}_{k}$ should really be different, aside from $\mathbb{Z}_{2}$ symmetry, which would be characterized by having different signs for $r$ in the bulk spaces, then the embeddings in (2.3) must differ in more than a sign. Especially equation (2.8) should look differently for $k=1$ resp. $k=2$.

Since $\mathbb{Z}_{2}$ symmetry would change equation (1.14) only by a factor 2 , our assumption that the branes are boundaries of a connected domain does not constitute a lack of generality. 


\section{The field equations}

Let us first summarize a few of our conventions. Covariant derivatives are always full tensors, i.e., for a map

$$
\varphi: N \longrightarrow \mathcal{S}
$$

the Laplacian $\Delta \varphi^{A}$ is defined by

$$
\Delta \varphi^{A}=\bar{g}^{\alpha \beta} \varphi_{\alpha \beta}^{A}
$$

where $\varphi_{\alpha \beta}^{A}$ are second order covariant derivatives of $\varphi$ which are defined by

$$
\varphi_{\alpha \beta}^{A}=\varphi_{, \alpha \beta}^{A}-\bar{\Gamma}_{\alpha \beta}^{\gamma} \varphi_{\gamma}^{A}+\hat{\Gamma}_{B C}^{A} \varphi_{\alpha}^{B} \varphi_{\beta}^{C}
$$

where a comma indicates as usual ordinary partial derivatives.

Recall the Gauß formula

$$
y_{\alpha \beta}=-\sigma h_{\alpha \beta} \nu
$$

for a hypersurface $N \subset \mathcal{N}$ given by an embedding $y=y\left(\xi^{\alpha}\right)$, where $\sigma=$ $\langle\nu, \nu\rangle$, which implicitly defines the second fundamental form. For a brane, $\nu$ is spacelike, i.e., $\sigma=1$.

For a more detailed overview of our conventions we refer to [1, Section $2]$, where hypersurfaces in an albeit different setting are considered, but this shouldn't cause any difficulties.

Suppose now that the metric in the bulk and the embeddings of the boundary branes satisfy a variational principle, namely, that the first variation of the functional $I$ in (1.1) vanishes with respect to variations of the metric that have compact support in $\Omega \cup N_{1} \cup N_{2}$. Then, by first choosing variations with compact support in $\Omega$, we deduce that the bulk metric $\left(\tilde{g}_{a b}\right)$ is an Einstein metric

$$
G_{a b}+\Lambda \tilde{g}_{a b}=0
$$

and by looking at a general variation we obtain, after an integration by parts, as a free boundary condition for each brane, the so-called Israel junction 
conditions

$$
\begin{aligned}
h_{\alpha \beta}-\frac{1}{2} H \bar{g}_{\alpha \beta} & =\kappa\left(L_{\text {tot }} \bar{g}_{\alpha \beta}-2 \frac{\partial L_{\text {tot }}}{\partial \bar{g}^{\alpha \beta}}-\sigma \bar{g}_{\alpha \beta}\right) \\
& =\kappa\left(\hat{T}_{\alpha \beta}-\sigma \bar{g}_{\alpha \beta}\right),
\end{aligned}
$$

where

$$
\hat{T}_{\alpha \beta}=\sum_{k=0}^{k_{0}}{ }_{k} T_{\alpha \beta}+T_{\alpha \beta}
$$

is the total stress-energy tensor, $\left({ }_{k} T_{\alpha \beta}\right), 0 \leq k \leq k_{0}$, the stress-energy tensors of perfect fluids with constant $\omega_{k}, \omega_{k} \in \mathbb{Z}$, and $\left(T_{\alpha \beta}\right)$ the stress-energy tensor associated with the scalar fields, i.e., associated with the map $\varphi$

$$
T_{\alpha \beta}=\left(-\frac{1}{2}\|D \varphi\|^{2}-V\right) \bar{g}_{\alpha \beta}+g_{A B} \varphi_{\alpha}^{A} \varphi_{\beta}^{B} .
$$

Since the bulk metric, which we now assume to be of the form (1.7), is Einstein, the left-hand side of (3.6) is divergence free, in view of the Codazzi equations, i.e., $\left(\hat{T}_{\alpha \beta}\right)$ is divergence free. Assuming in addition that each of the $\left({ }_{k} T_{\alpha \beta}\right)$ has vanishing divergence, then there must hold

$$
0=T_{\beta ; \alpha}^{\alpha}=\left(\Delta \varphi^{A}-\frac{\partial V}{\partial \varphi_{A}}\right) \frac{\partial \varphi_{A}}{\partial x^{\beta}}
$$

Though this does not necessarily imply that $\varphi$ satisfies the wave equation

$$
\Delta \varphi^{A}-\frac{\partial V}{\partial \varphi_{A}}=0
$$

the reverse is certainly true.

The Israel junction condition (3.6) is equivalent to

$$
h_{\alpha \beta}=\kappa\left(\hat{T}_{\alpha \beta}-\frac{1}{n} \hat{T} \bar{g}_{\alpha \beta}+\frac{\sigma}{n} \bar{g}_{\alpha \beta}\right) \text {, }
$$

where $\hat{T}=\hat{T}_{\alpha}^{\alpha}$.

To solve these equations let us look at the embeddings of the form (1.9) or (1.12). Both forms are equivalent as we shall see later and both have their advantages and disadvantages.

Let us consider the embedding (1.9), where $y=y\left(\tau, x^{i}\right)$ is parametrized in the conformal time gauge. The induced metric is then of the form (1.10) 
and we shall assume that the components of $\left(\hat{T}_{\alpha \beta}\right)$ only depend on $\tau$. Since $\left(\hat{T}_{\alpha \beta}\right)$ is derived from perfect fluids, we deduce

$$
\hat{T}_{0}^{0}=-\hat{\rho}, \quad \hat{T}_{i}^{\alpha}=\hat{p} \delta_{i}^{\alpha},
$$

where

$$
\hat{\rho}=\rho+\sum_{k=0}^{k_{0}} \rho_{k}, \quad \hat{p}=p+\sum_{k=0}^{k_{0}} p_{k}
$$

and

$$
\begin{gathered}
p_{k}=\frac{\omega_{k}}{n} \rho_{k}, \quad \omega_{k} \in \mathbb{Z}, \\
\rho=-\frac{1}{2}\|D \varphi\|^{2}+V,
\end{gathered}
$$

and

$$
p=-\frac{1}{2}\|D \varphi\|^{2}-V .
$$

Note that in the conformal time gauge

$$
-\|D \varphi\|^{2}=g_{A B} \dot{\varphi}^{A} \dot{\varphi}^{B} e^{-2 f} \equiv\|\dot{\varphi}\|^{2} e^{-2 f} .
$$

We do not distinguish different norms by notation, since it will be evident by their arguments how the norms are to be understood.

Due to our assumption that the $\left({ }_{k} T_{\alpha \beta}\right)$ are divergence free, we conclude

$$
\rho_{k}=\bar{\rho}_{k} e^{-\left(n+\omega_{k}\right) f},
$$

where $0 \leq \bar{\rho}_{k}$ is an integration constant, cf., e.g., [4, Lemma 0.2]. Hence we have

$$
\hat{\rho}=\rho+\sum_{k=0}^{k_{0}} \bar{\rho}_{k} \mathrm{e}^{-\left(n+\omega_{k}\right) f}=\rho+\sum_{k=0}^{k_{0}} \bar{\rho}_{k}(-r)^{-\left(n+\omega_{k}\right)} .
$$

We observe that this relation remains valid, if we parametrize the embedding with respect to $r$, the radial gauge, since $r=r(\tau)$ and $\frac{d r}{d \tau} \neq 0$, as we shall show.

Remark 3.1. We stipulate that $\omega_{0}=n$ and that formally it should always be present, its actual presence depending on the values of $\bar{\rho}_{0}$, namely, $\bar{\rho}_{0}>0$ or $\bar{\rho}_{0}=0$. Moreover, we assume $\omega_{k}<n$ for $1 \leq k$. 
So far, we haven't used the Israel junction condition. Combining (3.11), (3.12) and (3.19) we infer

$$
\begin{aligned}
h_{i j}=\frac{\kappa}{n}(\hat{\rho}+\sigma) \bar{g}_{i j} & =\frac{\kappa}{n}\left(\rho+\sigma+\sum_{k=0}^{k_{0}} \bar{\rho}_{k}(-r)^{-\left(n+\omega_{k}\right)}\right) \bar{g}_{i j} \\
& \equiv \frac{\kappa}{n}(\rho+\sigma+\mathcal{C}) \bar{g}_{i j}
\end{aligned}
$$

for the spatial components of the second fundamental form.

Let us now derive $h_{i j}$ from the Gauß formula in the radial gauge. In this gauge, $N$ is a graph in the variables $\left(r, x^{i}\right)$ and we suppose that $\Omega$ lies below the brane. In this case, the normal vector $\nu$ in the Gauß formula, which is supposed to point to the exterior of $\Omega$, must have a positive component with respect to the $t$ coordinate.

The induced metric $\left(\bar{g}_{\alpha \beta}\right)$ is given by (1.13), the tangent vector is equal to $y^{\prime}=\left(1, t^{\prime}, 0, \ldots, 0\right)$, and the exterior normal

$$
\nu=\left(\nu^{\alpha}\right)=\frac{\tilde{h}^{-\frac{1}{2}}}{\sqrt{1-\tilde{h}^{2}\left|t^{\prime}\right|^{2}}}\left(\tilde{h}^{2} t^{\prime}, 1,0, \ldots, 0\right) .
$$

From (3.4) we conclude

$$
\begin{aligned}
y_{i j}^{t} & =-\bar{\Gamma}_{i j}^{0} t^{\prime}+\tilde{\Gamma}_{a b}^{t} y_{i}^{b} y_{j}^{c} \\
& =-h_{i j} \nu^{t}=-h_{i j} \frac{\tilde{h}^{-\frac{1}{2}}}{\sqrt{1-\tilde{h}^{2}\left|t^{\prime}\right|^{2}}},
\end{aligned}
$$

where $\left(\bar{\Gamma}_{\alpha \beta}^{\gamma}\right)$ resp. $\left(\tilde{\Gamma}_{a b}^{c}\right)$ are the Christoffel symbols in $N$ resp. $\mathcal{N}$, and we deduce further

$$
h_{i j}=\frac{\tilde{h} t^{\prime}}{\sqrt{\tilde{h}^{-1}-\tilde{h}\left|t^{\prime}\right|^{2}}} r^{-1} \bar{g}_{i j},
$$

and hence

$$
\frac{\tilde{h} t^{\prime}}{\sqrt{\tilde{h}^{-1}-\tilde{h}\left|t^{\prime}\right|^{2}}}=\frac{\kappa}{n}(\rho+\sigma+\mathcal{C}) r
$$

in view of $(3.20)$. 
Setting

$$
a=|\tilde{h}|^{2}\left|t^{\prime}\right|^{2}
$$

yields

$$
\begin{aligned}
\frac{1}{1-a} & =\frac{a}{1-a}+1=\frac{\kappa^{2}}{n^{2}}(\rho+\sigma+\mathcal{C})^{2} r^{2} \tilde{h}^{-1}+1 \\
& =\tilde{h}^{-1}\left(\tilde{h}+\frac{\kappa^{2}}{n^{2}}(\rho+\sigma+\mathcal{C})^{2} r^{2}\right)
\end{aligned}
$$

or

$$
\tilde{h}^{-1}(1-a)=\frac{1}{\tilde{h}+\left(\kappa^{2} / n^{2}\right)(\rho+\sigma+\mathcal{C})^{2} r^{2}}
$$

We need this relation for future reference.

Now, in the radial gauge, we have

$$
\begin{aligned}
\rho & =-\frac{1}{2}\|D \varphi\|^{2}+V=\frac{1}{2}\|\dot{\varphi}\|^{2} \frac{1}{\tilde{h}^{-1}-\tilde{h}\left|t^{\prime}\right|^{2}}+V \\
& =\frac{1}{2}\|\dot{\varphi}\|^{2} \frac{\tilde{h}}{1-a}+V
\end{aligned}
$$

from which, together with relation (3.24), we infer

$$
a(1-a)=\frac{\kappa^{2}}{4 n^{2}}\left(\|\dot{\varphi}\|^{2}+2 \tilde{h}^{-1}(1-a)(V+\sigma+\mathcal{C})\right)^{2} r^{2} \tilde{h}
$$

The solutions to this quadratic equation are

$$
\begin{aligned}
a= & \frac{\tilde{h}+2(V+\sigma+\mathcal{C})^{2}\left(\kappa^{2} / n^{2}\right) r^{2}+\|\dot{\varphi}\|^{2}\left(\kappa^{2} / n^{2}\right) \tilde{h} r^{2}(V+\sigma+\mathcal{C})}{2\left(\tilde{h}+\left(\kappa^{2} / n^{2}\right)(V+\sigma+\mathcal{C})^{2} r^{2}\right)} \\
& \pm \frac{\tilde{h} \sqrt{1-2\|\dot{\varphi}\|^{2}\left(\kappa^{2} / n^{2}\right)(V+\sigma+\mathcal{C}) r^{2}-\left(\kappa^{2} / n^{2}\right)\|\dot{\varphi}\|^{4} \tilde{h} r^{2}}}{2\left(\tilde{h}+\left(\kappa^{2} / n^{2}\right)(V+\sigma+\mathcal{C})^{2} r^{2}\right)} .
\end{aligned}
$$

The solution with the plus sign will be the right one in our case, as we shall show later. 
With that particular solution we further conclude

$$
\begin{aligned}
-\frac{1}{2}\|D \varphi\|^{2} & =\frac{1}{2}\|\dot{\varphi}\|^{2} \frac{\tilde{h}}{1-a} \\
& =\frac{\|\dot{\varphi}\|^{2}\left(\tilde{h}+\left(\kappa^{2} / n^{2}\right)(V+\sigma+\mathcal{C})^{2} r^{2}\right)}{1-\left(\kappa^{2} / n^{2}\right)\|\dot{\varphi}\|^{2}(V+\sigma+\mathcal{C}) r^{2}-D},
\end{aligned}
$$

where

$$
D=\sqrt{1-2\|\dot{\varphi}\|^{2}\left(\kappa^{2} / n^{2}\right)(V+\sigma+\mathcal{C}) r^{2}-\left(\kappa^{2} / n^{2}\right)\|\dot{\varphi}\|^{4} \tilde{h} r^{2}} .
$$

This quantity as well as $-\frac{1}{2}\|D \varphi\|^{2} r^{2 n}$ are of particular interest; we shall find solutions such that both terms are positive in the limit

$$
\lim _{r \rightarrow 0}-\frac{1}{2}\|D \varphi\|^{2} r^{2 n}>0,
$$

and

$$
\lim _{r \rightarrow 0} \sqrt{1-2\|\dot{\varphi}\|^{2}\left(\kappa^{2} / n^{2}\right)(V+\sigma+\mathcal{C}) r^{2}-\left(\kappa^{2} / n^{2}\right)\|\dot{\varphi}\| 4 \tilde{h} r^{2}}>0 .
$$

Moreover, we shall only be interested in solutions satisfying

$$
\lim _{r \rightarrow 0} V r^{2 n}=0 \text {. }
$$

Define the vector field

$$
\chi^{A}=\dot{\varphi}^{A} r^{-(n-1)}
$$

and let

$$
\begin{aligned}
\hat{h} & =\tilde{h} r^{4 n-2}=(-r)^{3 n-1} \tilde{h}(-r)^{n-1} \\
& =(-r)^{3 n-1}\left(m+\frac{2}{n(n+1)} \Lambda(-r)^{n+1}-\tilde{\kappa}(-r)^{n-1}\right) .
\end{aligned}
$$

With these definition we infer from (3.31)

$$
-\frac{1}{2}\|D \varphi\|^{2} r^{2 n}=\frac{\|\chi\|^{2}\left(\hat{h}+\left(\kappa^{2} / n^{2}\right)(V+\sigma+\mathcal{C})^{2} r^{4 n}\right)}{1-\left(\kappa^{2} / n^{2}\right)\|\chi\|^{2}(V+\sigma+\mathcal{C}) r^{2 n}-\hat{D}}
$$

where

$$
\hat{D}=\sqrt{1-2 \frac{\kappa^{2}}{n^{2}}\|\chi\|^{2}(V+\sigma+\mathcal{C}) r^{2 n}-\frac{\kappa^{2}}{n^{2}} \hat{h}\|\chi\|^{4}}
$$

Abbreviate the denominator as

$$
1-u-\sqrt{1-2 u-v},
$$


then we conclude

$$
\begin{array}{r}
(1-u-\sqrt{1-2 u-v})(1-u+\sqrt{1-2 u-v}) \\
=(1-u)^{2}-(1-2 u-v)=u^{2}+v
\end{array}
$$

and hence

$$
1-u-\sqrt{1-2 u-v}=\left(u^{2}+v\right)(1-u+\sqrt{1-2 u-v})^{-1} .
$$

By definition

and thus

$$
\begin{gathered}
v=\frac{\kappa^{2}}{n^{2}}\|\chi\|^{4} \hat{h}, \\
u^{2}=\frac{\kappa^{4}}{n^{4}}\|\chi\|^{4}(V+\sigma+\mathcal{C})^{2} r^{4 n}
\end{gathered}
$$

and we conclude

$$
u^{2}+v=\frac{\kappa^{2}}{n^{2}}\|\chi\|^{4}\left(\hat{h}+\frac{\kappa^{2}}{n^{2}}(V+\sigma+\mathcal{C})^{2} r^{4 n}\right)
$$

$$
-\frac{1}{2}\|D \varphi\|^{2} r^{2 n}=\frac{1-\left(\kappa^{2} / n^{2}\right)\|\chi\|^{2}(V+\sigma+\mathcal{C}) r^{2 n}+\hat{D}}{\left(\kappa^{2} / n^{2}\right)\|\chi\|^{2}}
$$

cf. (3.39).

By assumption $\mathcal{C}$ is at most of order $r^{-2 n}$, i.e.,

$$
\lim _{r \rightarrow 0} \mathcal{C} r^{2 n}=\bar{\rho}_{0},
$$

cf. Remark 3.1.

The term $\hat{D}$ in (3.39) has always to be positive, which will be the case near the singularity, if

$$
2 \frac{\kappa^{2}}{n^{2}}\|\chi(0)\|^{2} \bar{\rho}_{0}<1
$$

where $\|\chi(0)\|^{2}$ can be looked at either as an initial value or as a limit

$$
\lim _{r \rightarrow 0}\|\chi\|^{2}
$$

which will always be assumed to be positive.

Let us summarize what we have proved so far for $-\frac{1}{2}\|D \varphi\|^{2} r^{2 n}$, or equivalently, for $\rho r^{2 n}$ in a lemma

Lemma 3.1. Let $N$ be solution of the embedding problem, satisfying the estimates (3.33), (3.35), and (3.48), or more precisely, such that the corresponding term $\hat{D}$ in (3.39) is positive, then the quantity $\rho r^{2 n}$ is a smooth function in the (independent) variables $\left(r, \chi^{A}, \varphi^{B}\right)$ according to equation 
(3.46). The smoothness is even valid in a full neighbourhood, $-\epsilon<r<\epsilon$, of the singularity $r=0$, provided $\|\chi\|^{2}>0$.

Let us now transform the wave equation (3.10). We are still in the radial gauge and conclude from (3.3)

$$
\begin{aligned}
\Delta \varphi^{A} & =\bar{g}^{\alpha \beta} \varphi_{\alpha \beta}^{A}=\bar{g}^{00} \varphi_{00}^{A}+\bar{g}^{i j} \varphi_{i j}^{A} \\
& =\bar{g}^{00} \varphi_{, 00}^{A}-\bar{\Gamma}_{00}^{0} \varphi_{0}^{A} \bar{g}^{00}-\bar{g}^{i j} \bar{\Gamma}_{i j}^{0} \varphi_{0}^{A}+\hat{\Gamma}_{B C}^{A} \varphi_{0}^{B} \varphi_{0}^{C} \bar{g}^{00} \\
& =\ddot{\varphi}^{A} \bar{g}^{00}-\bar{\Gamma}_{00}^{0} \dot{\varphi}^{A} \bar{g}^{00}-\bar{g}^{i j} \bar{\Gamma}_{i j}^{0} \dot{\varphi}^{A},
\end{aligned}
$$

where

$$
\ddot{\varphi}^{A}=\frac{D}{d r} \dot{\varphi}^{A}
$$

is the covariant second derivative of $\left(\varphi^{A}\right)$ with respect to $r$.

Now,

$$
\begin{gathered}
-\bar{g}^{i j} \bar{\Gamma}_{i j}^{0}=\frac{n}{r} \bar{g}^{00}, \\
-\bar{\Gamma}_{00}^{0}=\frac{1}{2}\left(\tilde{h}^{-1}-\tilde{h}\left|t^{\prime}\right|^{2}\right)^{\prime} \bar{g}^{00}
\end{gathered}
$$

and

$$
\bar{g}^{00}=-\frac{1}{\tilde{h}^{-1}-\tilde{h}\left|t^{\prime}\right|^{2}}=-\frac{1}{\tilde{h}^{-1}(1-a)},
$$

in our previous notation.

Thus we have

$$
\Delta \varphi^{A}=\left(\ddot{\varphi}^{A}+\frac{n}{r} \dot{\varphi}^{A}-\frac{1}{2} \frac{\left(\tilde{h}^{-1}(1-a)\right)^{\prime}}{\tilde{h}^{-1}(1-a)} \dot{\varphi}^{A}\right) \bar{g}^{00}=\frac{\partial V}{\partial \varphi_{A}},
$$

or equivalently,

$$
\ddot{\varphi}^{A}=-\frac{n}{r} \dot{\varphi}^{A}+\frac{1}{2} \frac{\left(\tilde{h}^{-1}(1-a)\right)^{\prime}}{\tilde{h}^{-1}(1-a)} \dot{\varphi}^{A}-\frac{\partial V}{\partial \varphi_{A}}\left(\tilde{h}^{-1}(1-a)\right) .
$$

In view of (3.27) we have

$$
\tilde{h}^{-1}(1-a)=\frac{r^{4 n-2}}{\hat{h}+\left(\kappa^{2} / n^{2}\right)\left(\rho r^{2 n}+\sigma r^{2 n}+\mathcal{C} r^{2 n}\right)^{2}}
$$


and therefore

$$
\begin{aligned}
& \left(\tilde{h}^{-1}(1-a)\right)^{\prime}=\frac{(4 n-2) r^{4 n-2} r^{-1}}{\hat{h}+\left(\kappa^{2} / n^{2}\right)\left(\rho r^{2 n}+\sigma r^{2 n}+\mathcal{C} r^{2 n}\right)^{2}} \\
& -\frac{r^{4 n-2}\left(\hat{h}^{\prime}+2\left(\kappa^{2} / n^{2}\right)\left(\rho r^{2 n}+\sigma r^{2 n}+\mathcal{C} r^{2 n}\right)\left(\rho r^{2 n}+\sigma r^{2 n}+\left(\kappa^{2} / n^{2}\right) C r^{2 n}\right)^{\prime}\right)}{\left(\hat{h}+\left(\kappa^{2} / n^{2}\right)\left(\rho r^{2 n}+\sigma r^{2 n}+\mathcal{C} r^{2 n}\right)^{2}\right)^{2}},
\end{aligned}
$$

i.e.,

$$
\begin{aligned}
& \frac{\left(\tilde{h}^{-1}(1-a)\right)^{\prime}}{\tilde{h}^{-1}(1-a)}=(4 n-2) r^{-1} \\
& \quad-\frac{\hat{h}^{\prime}+2\left(\kappa^{2} / n^{2}\right)\left(\rho r^{2 n}+\sigma r^{2 n}+\mathcal{C} r^{2 n}\right)\left(\rho r^{2 n}+\sigma r^{2 n}+\left(\kappa^{2} / n^{2}\right) C r^{2 n}\right)^{\prime}}{\hat{h}+\left(\kappa^{2} / n^{2}\right)\left(\rho r^{2 n}+\sigma r^{2 n}+\mathcal{C} r^{2 n}\right)^{2}} .
\end{aligned}
$$

Finally, setting as before

$$
\chi^{A}=\dot{\varphi}^{A} r^{-(n-1)},
$$

such that

$$
\dot{\chi}^{A}=\ddot{\varphi}^{A} r^{-(n-1)}-(n-1) \dot{\varphi}^{A} r^{-n},
$$

we deduce from (3.56)

$$
\begin{aligned}
\dot{\chi}^{A}= & -\frac{\hat{h}^{\prime}+2\left(\kappa^{2} / n^{2}\right)\left(\rho r^{2 n}+\sigma r^{2 n}+\mathcal{C} r^{2 n}\right)\left(\rho r^{2 n}+\sigma r^{2 n}+\left(\kappa^{2} / n^{2}\right) C r^{2 n}\right)^{\prime}}{\hat{h}+\left(\kappa^{2} / n^{2}\right)\left(\rho r^{2 n}+\sigma r^{2 n}+\mathcal{C} r^{2 n}\right)^{2}} \chi^{A} \\
& -\frac{\partial V}{\partial \varphi_{A}} \frac{r^{3 n-1}}{\hat{h}+\left(\kappa^{2} / n^{2}\right)\left(\rho r^{2 n}+\sigma r^{2 n}+\mathcal{C} r^{2 n}\right)^{2}} .
\end{aligned}
$$

Furthermore, as we shall prove in Lemma 4.1,

$$
\left(\rho r^{2 n}\right)^{\prime}=\frac{d}{d r}\left(\rho r^{2 n}\right)=2 n V r^{2 n-1},
$$

and hence, equation (3.62) can be written as

$$
\begin{aligned}
\dot{\chi}^{A}= & -\frac{\hat{h}^{\prime}+2\left(\kappa^{2} / n^{2}\right)\left(\rho r^{2 n}+\hat{\mathcal{C}}\right)\left(2 n V r^{2 n-1}+\hat{\mathcal{C}}^{\prime}\right)}{\hat{h}+\left(\kappa^{2} / n^{2}\right)\left(\rho r^{2 n}+\hat{\mathcal{C}}\right)^{2}} \chi^{A} \\
& -\frac{\partial V}{\partial \varphi_{A}} \frac{r^{3 n-1}}{\hat{h}+\left(\kappa^{2} / n^{2}\right)\left(\rho r^{2 n}+\hat{\mathcal{C}}\right)^{2}}
\end{aligned}
$$

where the term $\rho r^{2 n}$ has to be replaced with the help of (3.46), and where we used the abbreviation

$$
\hat{\mathcal{C}}=(\sigma+\mathcal{C}) r^{2 n}
$$


The equation (3.64) has the form

$$
\frac{D}{d r} \chi^{A}=F^{A}(r, \varphi, \chi),
$$

where $F=\left(F^{A}\right)$ is a smooth vector field which is defined in $(-\epsilon, \epsilon) \times \mathcal{S} \times$ $T^{1,0}(\mathcal{S})$ as long as $\|\chi\|^{2}>0$, the right-hand side of (3.46) is well defined and positive, and $\epsilon$ is small.

A solution $\chi(r)$ is considered to be a vector field over the map $\varphi(r)$, i.e., if $\varphi(r)$ is not known a priori, then the system is incomplete and has to be completed before a solution can be found, cf. equation (3.72).

Lemma 3.2. The field equation (3.66) has to be satisfied by any solution of the embedding problem, that satisfies equations (3.10), (3.24) and the specified assumptions on radial symmetry, with $\chi=\dot{\varphi} r^{-(n-1)}$, provided

$$
\begin{gathered}
\|\chi(0)\|^{2}=\lim _{r \rightarrow 0}\|\chi\|^{2}>0, \\
\lim _{r \rightarrow 0} \rho r^{2 n}>\bar{\rho}_{0}, \\
\lim _{r \rightarrow 0} V r^{2 n}=0,
\end{gathered}
$$

and the estimate (3.48) is valid. The last assumption is redundant, if $\bar{\rho}_{0}=0$.

Proof. In the derivation of the field equation (3.66) we had at one point to make a choice, namely, to pick one of two possible solutions in (3.30). If the above assumptions would imply that the choice with the plus sign is the only possible, then the lemma would be proved.

We shall compare the limit in (3.68) depending on the possible choices in (3.30). If we choose the plus sign, then the term $-\frac{1}{2}\|D \varphi\|^{2} r^{2 n}$ has been explicitly expressed in (3.46) and, if we denote its limit by $\rho_{+}$, we get

$$
\rho_{+}=\frac{1-\left(\kappa^{2} / n^{2}\right)\|\chi(0)\|^{2} \bar{\rho}_{0}+\sqrt{1-2\left(\kappa^{2} / n^{2}\right)\|\chi(0)\|^{2} \bar{\rho}_{0}}}{\left(\kappa^{2} / n^{2}\right)\|\chi(0)\|^{2}} .
$$

In view of the condition (3.48), the estimate $\rho_{+}>\bar{\rho}_{0}$ is valid, as one easily checks.

On the other hand, if the corresponding quantity $\rho_{-}$is derived, we obtain

$$
\rho_{-}=\frac{\left(\kappa^{2} / n^{2}\right)\|\chi(0)\|^{2} \bar{\rho}_{0}}{1-\left(\kappa^{2} / n^{2}\right)\|\chi(0)\|^{2} \bar{\rho}_{0}+\sqrt{1-2\left(\kappa^{2} / n^{2}\right)\|\chi(0)\|^{2} \bar{\rho}_{0}}} \bar{\rho}_{0},
$$

where the quotient is less than or equal to 1 , in view of (3.48).

Let us conclude this section with the following theorem 
Theorem 3.1. The first order system

$$
\begin{aligned}
& \dot{\chi}^{A}=F^{A}(r, \varphi, \chi)-\hat{\Gamma}_{B C}^{A} \chi^{B} \chi^{C} r^{n-1} \\
& \dot{\varphi}^{B}=\chi^{B} r^{n-1}
\end{aligned}
$$

where the dot indicates an ordinary derivative with respect to $r$, has a smooth solution $\left(\chi^{A}, \varphi^{B}\right)$ in a maximal interval $\left(-\epsilon_{1}, \epsilon_{2}\right), \epsilon_{i}>0$, for any initial values $\chi(0), \varphi(0)$, provided $\chi(0)$ is such that $\|\chi(0)\|^{2}>0$ and the estimate (3.48) is satisfied. Using the relation (3.70) as the definition of $\rho_{+}$, we also define $\rho_{+}$for the solution of (3.72).

The first equation in (3.72) is just a rephrasing of (3.66). The existence of a smooth solution of the initial value problem follows from the general existence theorem for integral curves. Notice also that the assumption $\|\chi(0)\|^{2}>0$ can be satisfied, since the metric of $\mathcal{S}$ has at least one positive eigenvalue.

We shall show in the next section that, if we restrict the solution from Theorem 3.1 to $\{r<0\}$, then $\varphi$ is a solution of our embedding problem.

\section{Existence of a global solution of the embedding problem}

By a "global solution" of the embedding problem we mean a solution given by Theorem 3.1 that is a solution of the embedding problems in $\mathcal{N}$ resp. $\hat{\mathcal{N}}$, when restricted to $(-\epsilon, 0)$ resp. $(0, \epsilon), \epsilon>0$ small.

We shall prove that the solution of Theorem 3.1 has indeed the required properties, if $n$ and the $\omega_{k}$ are odd, since it satisfies

$$
\rho_{+}>\rho_{0}
$$

Let us first show that the solution in Theorem 3.1 is also a solution of the embedding problem in $\mathcal{N}$.

Look at the embedding in the conformal time gauge $x^{0}=\tau$, cf. (1.9) and (1.10), where now, however, the value $\tau=0$ should be mapped onto $r=-\delta$, $\delta>0$, i.e., we stay away from the singularity.

We shall show that this problem can be solved in a maximal interval $J=(a, b)$, with $0 \in J$, for given initial values at $\tau=0$. If we then choose as initial values those of the solution from Theorem 3.1 evaluated at $r=-\delta$, where of course $\delta<\epsilon$, then we shall conclude that the solution given by Theorem 3.1 and the actual solution of the embedding problem coincide. 
In view of the maximality of $J, b$ must then correspond to $r=0$ and the solution of Theorem 3.1, restricted to $(-\epsilon, 0)$, will indeed be a solution of the embedding problem in $\mathcal{N}$.

In the conformal time gauge the energy density $\rho$ can be expressed as

$$
\begin{aligned}
\rho & =-\frac{1}{2}\|D \varphi\|^{2}+V \\
& =\frac{1}{2}\|\dot{\varphi}\|^{2} e^{-2 f}+V,
\end{aligned}
$$

where we again use the same symbol $\|\cdot\|$ to denote different norms. A dot or a prime now indicates differentiation with respect to $\tau$.

The Israel junction conditions lead to the so-called modified Friedman equation

$$
\left|f^{\prime}\right|^{2}=\tilde{h}+\frac{\kappa^{2}}{n^{2}}(\rho+\sigma+\mathcal{C})^{2} \mathrm{e}^{2 f},
$$

cf. e.g., the derivation in $[4$, Section 1]. Here, $\mathcal{C}$ has the same meaning as before.

We have to find $f=f(\tau)$ and a map $\varphi$ from $N$ to $\mathcal{S}, \varphi=\varphi\left(\tau, x^{i}\right)$, such that the relations (4.3) and the wave equation (3.10) are satisfied. Since $\varphi$ is supposed to depend only on $\tau$, an easy exercise shows that

$$
\Delta \varphi^{A}=-\left(\ddot{\varphi}^{A}+(n-1) f^{\prime} \dot{\varphi}^{A}\right) e^{-2 f}
$$

where a dot now indicates covariant differentiation with respect to $\tau$. Hence we obtain as second requirement

$$
\ddot{\varphi}^{A}=-(n-1) f^{\prime} \dot{\varphi}^{A}-\frac{\partial V}{\partial \varphi_{A}} e^{2 f} .
$$

Setting

$$
\tilde{\chi}^{A}=\dot{\varphi}^{A} e^{(n-1) f}
$$

we conclude

$$
\dot{\tilde{\chi}}=-\frac{\partial V}{\partial \varphi_{A}} e^{(n+1) f}
$$

Now we can solve the pair of equations (4.3) and (4.5). Notice also that $f^{\prime}$ has to be negative. 
Theorem 4.1. The equations (4.3) and (4.5) have a smooth solution $\left(f, \varphi^{A}\right)$ in the interval $(-\epsilon, 0]$ for small $\epsilon>0$, with arbitrary initial values

$$
\begin{aligned}
f(0) & =\log (\delta), \\
\varphi^{A}(0) & =\varphi_{0}^{A}, \\
\dot{\varphi}^{A}(0) & =\xi^{A},
\end{aligned}
$$

provided $\|\xi\|^{2}>0$.

Proof. Consider the first order system

$$
\begin{aligned}
\dot{\tilde{\chi}} & =-\frac{\partial V}{\partial \varphi_{A}} e^{(n+1) f}-\hat{\Gamma}_{B C}^{A} \tilde{\chi}^{B} \tilde{\chi}^{C} \mathrm{e}^{-(n-1) f}, \\
\dot{\varphi}^{B} & =\tilde{\chi}^{B} \mathrm{e}^{-(n-1) f}, \\
f^{\prime} & =-\sqrt{\tilde{h}+\left(\kappa^{2} / n^{2}\right)(\rho+\sigma+\mathcal{C})^{2} e^{2 f}},
\end{aligned}
$$

where all derivatives are ordinary derivatives with respect to $\tau$, with initial values in (4.8), except that now

$$
\tilde{\chi}^{A}(0)=\xi^{A} \delta^{n-1} .
$$

This system has a smooth solution on $(-\epsilon, 0]$ for small $\epsilon>0$.

The requirement $\|\xi\|^{2}>0$ can be satisfied, since $\left(g_{A B}\right)$ is supposed to have at least one positive eigenvalue.

Now, to avoid confusion denote the solution from Theorem 4.1 by $\tilde{\varphi}^{A}$ and the solution from Theorem 3.1 by $\varphi^{A}$ and $\chi^{A}$.

We want to choose the initial values in (4.8) such that

$$
\tilde{\varphi}^{A}(0)=\varphi^{A}(-\delta)
$$

and

$$
\frac{d}{d r} \tilde{\varphi}^{A}(-\delta)=\chi^{A}(-\delta)(-\delta)^{n-1}
$$

where we observe that $\tilde{\varphi}$ can also be viewed as depending on $r$, since $f^{\prime}$ is negative. 
We have

$$
\begin{aligned}
\frac{d}{d r} \tilde{\varphi}^{A} & =\frac{d}{d \tau} \tilde{\varphi} \frac{d \tau}{d r}=-\frac{d}{d \tau} \tilde{\varphi} \frac{e^{-f}}{f^{\prime}} \\
& =\frac{d}{d \tau} \tilde{\varphi} \frac{e^{-} f}{\sqrt{\tilde{h}+\left(\kappa^{2} / n^{2}\right)(\rho+\sigma+\mathcal{C})^{2} e^{2 f}}},
\end{aligned}
$$

in view of (4.9). The requirement (4.12) is equivalent to

$$
\chi^{A}(-\delta)(-\delta)^{n-1}=\frac{\xi^{A} e^{-f}}{\sqrt{\tilde{h}+\left(\kappa^{2} / n^{2}\right)(\rho+\sigma+\mathcal{C})^{2} e^{2 f}}} .
$$

Obviously, we can choose

$$
\xi^{A}=\lambda \chi^{A}(-\delta), \quad \lambda \in \mathbb{R},
$$

so that we only need to fine-tune $\lambda$ such that

$$
\lambda^{2}=\delta^{2 n}\left(\tilde{h}+\frac{\kappa^{2}}{n^{2}}(\rho+\sigma+\mathcal{C})^{2} \delta^{2}\right) .
$$

The quantity $\rho$ is equal to

$$
\begin{aligned}
\rho & =\frac{1}{2}\|\dot{\varphi}\|^{2} e^{-2 f}+V \\
& =\frac{1}{2} \lambda^{2}\|\chi(-\delta)\|^{2} \delta^{-2}+V .
\end{aligned}
$$

Combining (4.16) and (4.17) we conclude

$$
\begin{aligned}
\lambda^{2}= & 2 \delta^{-2(n-1)}\|\chi(-\delta)\|^{-4} \frac{n^{2}}{\kappa^{2}}\left(1-(V+\sigma+\mathcal{C}) \delta^{2 n} \frac{\kappa^{2}}{n^{2}}\|\chi(-\delta)\|^{2}\right. \\
& \left.+\sqrt{1-2(V+\sigma+\mathcal{C}) \delta^{2 n} \frac{k^{2}}{n^{2}}\|\chi(-\delta)\|^{2}-\frac{k^{2}}{n^{2}} \delta^{4 n-2}\|\chi(-\delta)\|^{4} \tilde{h}}\right) .
\end{aligned}
$$

Of course, the power law integration constants are the same for both solutions, especially $\bar{\rho}_{0}$. Looking at equation (3.70), and observing that (3.48) is valid, we conclude, that there exists $\delta_{0}>0$, such that the relation (4.18) can be satisfied for any $0<\delta<\delta_{0}$ with $\lambda=\lambda(\delta) \in \mathbb{R}$. 
Let us now determine the value of $\rho \delta^{2 n}$ for our solution. Combining (4.17) and (4.18) we immediately deduce that

$$
\lim _{\delta \rightarrow 0} \rho \delta^{2 n}=\rho_{+},
$$

where $\rho_{+}$is the quantity that belongs to the solution from Theorem 3.1, i.e., $\rho_{+}>\bar{\rho}_{0}$. Hence Lemma 3.2 is applicable and the pair $\left(\bar{\chi}^{A}, \tilde{\varphi}^{B}\right)$, where

$$
\bar{\chi}^{A}=\frac{d}{d r} \tilde{\varphi}^{A} r^{-(n-1)},
$$

satisfies the field equation (3.66), or equivalently, the system (3.72).

Since $\left(\bar{\chi}^{A}, \tilde{\varphi}^{B}\right)$ coincide with $\left(\chi^{A}, \varphi^{B}\right)$ at $r=-\delta$, we deduce

$$
\left(\chi^{A}, \varphi^{B}\right)=\left(\bar{\chi}^{A}, \tilde{\varphi}^{B}\right)
$$

in a neighbourhood of $r=-\delta$.

Now, define

$$
M=\left\{r \in(-\epsilon, 0): \tilde{\varphi}^{A}(r)=\varphi^{A}(r)\right\}
$$

Since $M$ is not empty, and due to the assumption, that $\tilde{\varphi}^{A}$ should be defined in a maximal interval, we easily deduce that $M=(-\epsilon, 0)$, i.e., the solution given by Theorem 3.1 is indeed a physical solution in $\mathcal{N}$.

Now, assume that $n$ and the $\omega_{k}$ are odd — we haven't used these assumptions yet, then, looking at the definition of the vector field $F^{A}(r, \varphi, \chi)$ in (3.64), we see that, had we considered the first variation of the action integral in $\hat{\mathcal{N}}$ with all the subsequent derivations, we would have obtained the identical vector field $F^{A}$.

Hence, the preceding arguments, valid in $\mathcal{N}$, also hold in $\hat{\mathcal{N}}$, and we conclude further that the solution of Theorem 3.1, restricted to $(0, \epsilon)$, is also a physical solution in $\hat{\mathcal{N}}$.

Since the transformation

$$
\left(r, t, x^{i}\right) \longrightarrow\left(r,-t, x^{i}\right)
$$

is an isometry in $\mathcal{N}$ as well as $\hat{\mathcal{N}}$, the previous considerations are valid for upper branes as well as lower branes, since the isometry in (4.23) maps an upper brane to a lower brane and vice versa.

Branes that are given by an embedding of the form

$$
y\left(r, x^{i}\right)=\left(r, t(r), x^{i}\right)
$$

can be easily distinguished, as being an upper or lower brane, by looking at the equation satisfied by $t^{\prime}$. 
An upper brane has to satisfy equation (3.24), while, in case of a lower brane, the equation looks like

$$
\frac{\tilde{h} t^{\prime}}{\sqrt{\tilde{h}^{-1}-\tilde{h}\left|t^{\prime}\right|^{2}}}=-\frac{\kappa}{n}(\rho+\sigma+\mathcal{C}) r
$$

These equations are identical in $\mathcal{N}$ resp. $\hat{\mathcal{N}}$.

Thus, let us express $t^{\prime}$ in our case, where $N \subset \mathcal{N}$ is an upper brane, by combining (3.24) and (3.26) to deduce

$$
t^{\prime}=\frac{\kappa}{n} \tilde{h}^{-1} \frac{(\rho+\sigma+\mathcal{C}) r}{\sqrt{\tilde{h}+\left(\kappa^{2} / n^{2}\right)(\rho+\sigma+\mathcal{C})^{2} r^{2}}},
$$

or equivalently,

$$
t^{\prime}=-\frac{\kappa}{n} \frac{1}{\tilde{h} r^{n-1}} \frac{\left(\rho r^{2 n}+\hat{\mathcal{C}}\right) r^{n-1}}{\sqrt{\hat{h}+\left(\kappa^{2} / n^{2}\right)\left(\rho r^{2 n}+\hat{\mathcal{C}}\right)^{2}}},
$$

cf. the definition in (3.65).

Since $t=t(r)$ is a smooth function in $(-\epsilon, \epsilon)$ and the right-hand side of (4.27) has constant sign in $\{r \neq 0\}$, this implies that the brane $\hat{N} \subset \hat{\mathcal{N}}$ that corresponds to the big bang part of the global solution must be a lower brane.

Let us summarize this observation in

Remark 4.1. Let $(N, \hat{N})$ be a global solution of the embedding problem, then the branes $N, \hat{N}$ have opposite positions, i.e., if $N$ is an upper brane, then $\hat{N}$ is a lower brane, and vice versa.

Remark 4.2. Let us emphasize that even in the general case, i.e., without assuming that $n$ and the $\omega_{k}$ are odd, $t=t(r)$ is of class $C^{\infty}((-\epsilon, 0])$, since this is the case for $\varphi^{A}$ and $\chi^{A}$, as well as $\rho r^{2 n}$, because $\rho r^{2 n}$ satisfies the differential equation

$$
\frac{d}{d r}\left(\rho r^{2 n}\right)=2 n V r^{2 n-1}
$$

as we shall prove in

Lemma 4.1. Let $\varphi^{A}$ be a solution of the embedding problem, then $\rho r^{2 n}$ satisfies (4.28). 
Proof. We choose the conformal time gauge. $\left(T_{\alpha \beta}\right)$ is a divergence free perfect fluid stress-energy tensor, hence $\rho$ satisfies

$$
\dot{\rho}=-n(\rho+p) f^{\prime}=-2 n(\rho-V) f^{\prime},
$$

since

$$
\rho+p=-\|D \varphi\|^{2}=2(\rho-V),
$$

where dot or prime indicate differentiation with respect to $\tau$.

Hence

$$
\frac{d \rho}{d r}=\dot{\rho} \frac{1}{r f^{\prime}}=-2 n(\rho-V) r^{-1}
$$

and

$$
\frac{d}{d r}\left(\rho r^{2 n}\right)=2 n V r^{2 n-1}
$$

Remark 4.3. The brane, the existence of which we have just proved, is of class $(B)$ with $\tilde{\gamma}=2 n-1$, i.e.,

$$
\lim _{\tau \rightarrow b}\left|f^{\prime}\right|^{2} \mathrm{e}^{2 \tilde{\gamma} f}=\tilde{m}>0
$$

where $b \in \overline{\mathbb{R}}$ is such that

$$
\lim _{\tau \rightarrow b} \mathrm{e}^{f}=0 .
$$

Hence, $b$ is finite and we may choose $b=0$.

Proof. See [4, Definition 0.1] for the definition of class $(B)$ spacetimes. The conclusion " $b$ finite" is proved in [2, Lemma 3.1], so that it remains to verify (4.33).

From (4.3) we conclude

$$
\left|f^{\prime}\right|^{2} \mathrm{e}^{2 \tilde{\gamma} f}=\hat{h}+\frac{\kappa^{2}}{n^{2}}\left(\rho r^{2 n}+\hat{\mathcal{C}}\right)^{2},
$$

i.e.,

$$
\lim _{\tau \rightarrow b}\left|f^{\prime}\right|^{2} \mathrm{e}^{2 \tilde{\gamma} f}=\frac{\kappa^{2}}{n^{2}}\left(\lim _{r \rightarrow 0} \rho r^{2 n}+\bar{\rho}_{0}\right)^{2}
$$




\section{The reflection method}

In case $\mathcal{S}$ is locally symmetric and $V$ invariant in the neighbourhood of a point $p \in \mathcal{S}$ with respect to the geodesic symmetry centered at $p$, then the big bang part $\hat{N}$ of the global solution can be obtained by an odd reflection of $N$, namely, define

$$
\hat{y}\left(r, \hat{t}(r), x^{i}\right)=\left(r,-t(-r), x^{i}\right)
$$

for $r>0$, where we have normalized the globally defined function $t(r)$, representing the embedding of $N$, if $r<0$, by setting $t(0)=0$, which can be easily achieved by adding a constant.

We want to prove that

$$
\hat{t}(r)=t(r), \quad r>0,
$$

and hence conclude that $t$ is an odd function.

Theorem 5.1. Let $n$ and $\omega_{k}, 0 \leq k \leq k_{0}$, be odd and let $\mathcal{S}$ be a locally symmetric, semi-Riemannian space such that its metric $\left(g_{A B}\right)$ has at least one positive eigenvalue. Furthermore, assume that there is a point $p \in \mathcal{S}$ such that $V \in C^{\infty}(\mathcal{S})$ is invariant in a neighbourhood of $p$ with respect to the geodesic symmetry centered at $p$.

If we choose in the global existence theorem, Theorem 3.1, the initial condition $\varphi(0)=p$, then the quantity $\rho r^{2 n}$ can be looked at as an even function in a neighbourhood of $r=0$.

Moreover, introducing Riemannian normal coordinates around $p$, such that $\varphi^{A}(0)=0$, the globally defined scalar fields map $\left(\varphi^{A}\right)$ is an odd map in a neighbourhood of $r=0$.

The function $t=t(r)$ is odd as well in a neighbourhood of $r=0$.

Proof. After the introduction of Riemannian normal coordinates around $p$, such that $p$ corresponds to $p=0$, the geodesic symmetry centered in $p$ is nothing but odd reflection

$$
\varphi^{A} \longrightarrow-\varphi^{A}
$$

i.e., the invariance property of $V$ can be expressed by saying that $V$ is even in a neighbourhood of $\varphi=0$.

The vector field $F^{A}(r, \varphi, \chi)$ is defined in (3.64). Let us define a variant of $F^{A}$

$$
\Phi^{A}(r, u, \varphi, \chi)
$$

by treating the term $\rho r^{2 n}$ in $F^{A}$ as a new variable $u$. 
We found our solution of the global embedding problem as a solution of the first order system (3.72), cf. Theorem 3.1.

Since the quantity $\rho r^{2 n}$, evaluated for that particular solution, is smooth and satisfies

$$
\frac{d}{d r}\left(\rho r^{2 n}\right)=2 n V r^{2 n-1},
$$

the solution from Theorem 3.1 will also be a solution of the modified system

$$
\begin{aligned}
\dot{\chi}^{A} & =\Phi^{A}(r, u, \varphi, \chi)-\hat{\Gamma}_{A B}^{A} \chi^{B} \chi^{C} r^{n-1}, \\
\dot{\varphi} & =\chi^{B} r^{n-1}, \\
\dot{u} & =2 n V r^{2 n-1}
\end{aligned}
$$

to the given initial values.

Notice that we choose

$$
u(0)=\lim _{r \rightarrow 0} \rho r^{2 n}
$$

Let us denote the global solution of (5.6) by $\varphi^{A}(r), \chi^{B}(r)$, and $u(r)$.

Now, define for $r>0$ the reflected quantities

$$
\begin{aligned}
\tilde{\chi}^{A}(r) & =\chi^{A}(-r), \\
\tilde{\varphi}^{B}(r) & =-\varphi^{B}(-r), \\
\tilde{u}(r) & =u(-r),
\end{aligned}
$$

then

$$
\begin{aligned}
\dot{\tilde{\chi}}^{A}(r) & =-\dot{\chi}^{A}(-r), \\
\dot{\tilde{\varphi}}^{B}(r) & =\dot{\varphi}^{B}(-r), \\
\dot{\tilde{u}}(r) & =-\dot{u}(-r) .
\end{aligned}
$$

$\tilde{u}, \tilde{\varphi}, \tilde{\chi}$ are smooth in $[0, \epsilon)$ and in $r=0$ they have the same values as $u$, $\varphi, \chi$. If we can prove that $(\tilde{u}, \tilde{\varphi}, \tilde{\chi})$ satisfy the first order system in (5.6) in $(0, \epsilon)$, the uniqueness of the solution will imply that they coincide with $(u, \varphi, \chi)$, and the theorem will be proved, in view of (4.27).

Now, for a locally symmetric space, the Christoffel symbols $\hat{\Gamma}_{B C}^{A}$ are odd, i.e.,

$$
\hat{\Gamma}_{B C}^{A}(-\varphi)=-\hat{\Gamma}_{B C}^{A}(\varphi)
$$

in Riemannian normal coordinate, and one easily checks that

$$
\Phi^{A}(r, \tilde{u}, \tilde{\varphi}, \tilde{\chi})=-\Phi^{A}\left(-r, u(-r), \varphi^{A}(-r), \chi^{A}(-r)\right) .
$$

Hence $(\tilde{u}, \tilde{\varphi}, \tilde{\chi})$ are solutions and the theorem is proved. 


\section{Colliding branes}

Consider a domain $\Omega \subset \mathcal{N}$ bounded by two branes $N_{1}$ and $N_{2}$. If $N_{1}$ is the upper brane and $N_{2}$ the lower, and if, after having solved the global embedding problem for each single brane, resulting in branes $\hat{N}_{1}$ and $\hat{N}_{2}$, we would want to describe the configuration in $\hat{\mathcal{N}}$ similarly as in $\mathcal{N}$, namely, that $\hat{N}_{i}$ are boundary branes of a domain $\hat{\Omega}$, then this is only possible, if $N_{1}$ and $N_{2}$ collide at the singularity.

Lemma 6.1. Suppose that each single brane $N_{i}$ can be considered as the big crunch part of a globally defined solution, then the branes $\hat{N}_{i}$ are boundary branes of domain $\hat{\Omega} \subset \hat{\mathcal{N}}$, if and only if the branes $N_{i}$ collide at the singularity.

Proof. Let us write the global solutions as globally defined graphs

$$
t=u_{i}\left(r, x^{i}\right), \quad r \in(-\epsilon, \epsilon),
$$

with smooth functions $u_{i}$.

Without loss of generality let us assume that $N_{1}$ is an upper brane and $N_{2}$ a lower, i.e., we have

$$
u_{1} \geq u_{2}, \quad r<0 \text {. }
$$

However, in $\hat{\mathcal{N}}$ the branes reverse positions and therefore we have

$$
u_{1} \leq u_{2}, \quad r>0
$$

and hence $u_{1}(0)=u_{2}(0)$.

On the other hand, assume that $u_{1}(0)=u_{2}(0)$. Since $\Omega$ can be written as

$$
\Omega=\left\{\left(r, t, x^{i}\right): u_{1}(r)>t>u_{2}(r), r<0\right\},
$$

we immediately see that

$$
\hat{\Omega}=\left\{\left(r, t, x^{i}\right): u_{1}(r)<t<u_{2}(r), r>0\right\} .
$$

Remark 6.1. If the assumptions of part (iii) of Theorem 1.1 are satisfied for two boundary branes, then it can be easily achieved that they collide at the singularity by translating one brane in the $t$-direction. 


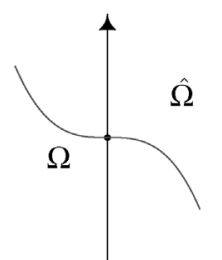

A globally defined single brane.

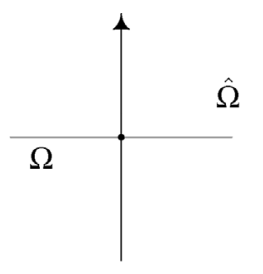

The totally geodesic brane $\{t=$ const $\}$.

Below are two examples of colliding branes. The $t$-axis represents the black (white) hole singularity.
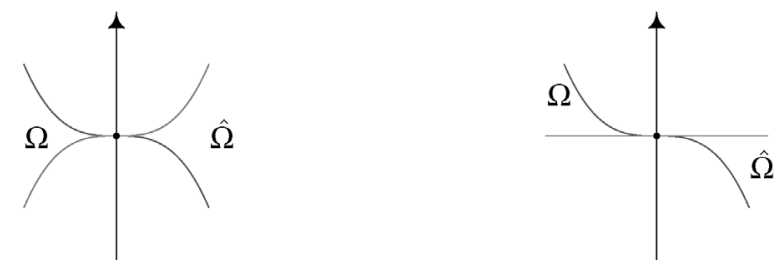

From a physical and geometrical point of view it would be desirable to find sufficient conditions guaranteeing that the branes collide only at the singularity.

Theorem 6.1. Let $N_{i}$ be two branes in the black hole region of $\mathcal{N}$, which are boundary branes of a domain $\Omega$, where we assume that $N_{1}$ is the upper brane and $\mathrm{N}_{2}$ the lower brane. Parametrizing both branes with respect to $r<0$, we can write each brane as a graph

$$
t=u_{i}(r)
$$

such that

$$
u_{1} \geq u_{2} .
$$

In view of the Israel junction conditions, the $u_{i}$ satisfy the equations

$$
\frac{\tilde{h} u_{i}^{\prime}}{\sqrt{\tilde{h}^{-1}-\tilde{h}\left|u_{i}^{\prime}\right|^{2}}}= \pm \frac{\kappa}{n}\left(\rho_{i}+\sigma_{i}+\mathcal{C}_{i}\right) r,
$$

$c f .(4.25)$, where the plus sign corresponds to $u_{1}$ and the minus sign to $u_{2}$.

Suppose, moreover, that the equation

$$
\left(\rho_{1}+\sigma_{1}+\mathcal{C}_{1}\right)+\left(\rho_{2}+\sigma_{2}+\mathcal{C}_{2}\right)=0
$$

cannot be valid in the interval $(-a, 0)$, then the only possible collision point in $(-a, 0]$ of the branes is $r=0$. 
Proof. This is an immediate consequence of Rolle's theorem.

\section{Maximal extension of the branes}

We now consider branes without assuming that $n$ or the $\omega_{k}$ 's are odd, and look at a solution given by Theorem 3.1, which, when restricted to $\{r<0\}$, is defined on a maximal interval $\left\{r_{1}<r \leq 0\right\}$, with $-r_{1}<r_{0}$, where $r_{0}$ is the black hole radius.

Notice that the field equations (3.72) are probably only valid in a much smaller interval $\{-\epsilon<r \leq 0\}$, since the quantity $\hat{D}$ in (3.39) may no longer be positive outside the smaller interval.

We want to prove that we may choose $r_{1}=-r_{0}$, if $\mathcal{S}$ is Riemannian and complete, and if in addition $\rho$ is bounded from below

$$
\rho \geq-c, \quad c>0
$$

which is slightly better than requiring that the potential $V$ is bounded from below.

Pick $\bar{r} \in\left(r_{1}, 0\right),|\bar{r}|<1$, and switch to the conformal time gauge. Set

$$
\delta=\log (-\bar{r})<0,
$$

so that the brane is defined on a maximal interval $J=\{a<\tau \leq \delta\}, a \in \overline{\mathbb{R}}$.

The relevant terms are now $\left(f, \varphi^{A}\right)$ satisfying equations (4.3) and (4.5), or the equivalent one (4.7), which is

$$
\dot{\tilde{\chi}}=-\frac{\partial V}{\partial \varphi_{A}} \mathrm{e}^{(n+1) f}
$$

where

$$
\tilde{\chi}^{A}=\dot{\varphi}^{A} \mathrm{e}^{(n-1) f} .
$$

All derivatives are covariant derivatives with respect to $\tau$, " $D / d \tau$ ".

We certainly will hit a coordinate singularity, if we approach the horizon, i.e., $\mathrm{e}^{f}$ will always be bounded from below and above by positive constants. The quantity $\mathcal{C}$ in (4.3) is likewise uniformly bounded.

For convenience let us also change from $\tau$ to $-\tau$, so that $J=[\delta, a), 0<\delta$; notice that this switch does not effect (7.3) and (7.4), because these equations are tensor equations. The only difference is that $f^{\prime}>0$. 
Lemma 7.1. Let $J=[\delta, a)$ and assume that horizon is not reached, then $a<\infty$.

Proof. Since $f^{\prime}>0$, we deduce from (4.3)

$$
f^{\prime} \geq \sqrt{\tilde{h}} \geq c>0
$$

since the horizon is not reached. Hence, $a<\infty$, for otherwise $f$ would tend to infinity and so would $r=-e^{f}$.

Lemma 7.2. If $a<\infty$ and (7.1) is valid, then

$$
\|\dot{\varphi}\| \leq c \quad \forall \tau \in J
$$

$\varphi(J) \subset \mathcal{S}$ is precompact, and hence

$$
|V| \leq c \quad \text { and } \quad|\rho| \leq c .
$$

Proof. (i) We use the relation (7.3) to conclude

$$
\begin{aligned}
V(\delta)-V(\tau) & =-\int_{\delta}^{\tau} V^{\prime}=-\int_{\delta}^{\tau} \frac{\partial V}{\partial \varphi_{A}} \dot{\varphi}^{A} \\
& =\int_{\delta}^{\tau}\left(\frac{1}{2}\|\tilde{\chi}\|^{2}\right)^{\prime} \mathrm{e}^{-2 n f} \\
& =\left.\frac{1}{2}\|\tilde{\chi}\|^{2} \mathrm{e}^{-2 n f}\right|_{\delta} ^{\tau}+n \int_{\delta}^{\tau}\|\tilde{\chi}\|^{2} f^{\prime} .
\end{aligned}
$$

Now, in view of (7.5) and the definition of $\rho$, we infer

$$
c \int_{\delta}^{\tau}\|\dot{\varphi}\|^{2}+\rho(\tau) \leq \rho(\delta)
$$

and hence

$$
\int_{\delta}^{\tau}\|\dot{\varphi}\|^{2} \leq c \quad \forall \tau \in J
$$

Moreover, because of equation (4.29) there holds

$$
\dot{\rho}=-n\left(\frac{1}{2}\|\dot{\varphi}\|^{2} \mathrm{e}^{-2 f}\right) f^{\prime} \leq 0,
$$

i.e., $\rho$ is bounded from above and thus we have

$$
|\rho| \leq c \quad \forall \tau \in J .
$$


(ii) Next we want to show that $\varphi: J \rightarrow \mathcal{S}$ is uniformly Hölder continuous. Let $d$ be the Riemannian distance in $\mathcal{S}$, then, for any $\delta \leq \tau \leq \tau^{\prime}<a$

$$
\begin{aligned}
d\left(\varphi(\tau), \varphi\left(\tau^{\prime}\right)\right) & \leq \int_{\tau}^{\tau^{\prime}}\|\dot{\varphi}\| \leq\left|\tau^{\prime}-\tau\right|^{\frac{1}{2}}\left(\int_{\tau}^{\tau^{\prime}}\|\dot{\varphi}\|^{2}\right)^{\frac{1}{2}} \\
& \leq c\left|\tau^{\prime}-\tau\right|^{1 / 2}
\end{aligned}
$$

i.e.,

$$
\varphi \in C^{0,1 / 2}(\bar{J}, \mathcal{S})
$$

and $\varphi$ can be extended to $\bar{J}$ as a Hölder continuous function, since $\mathcal{S}$ is complete. But $\bar{J}$ is compact, and hence $\varphi(\bar{J})$ is compact, and we conclude that $V \circ \varphi$ is uniformly bounded on $J$, and hence $\|\dot{\varphi}\|$, in view of (7.12).

But with these a priori estimates it follows immediately that equations (4.3) and (4.5) can be solved on a larger interval, i.e., $J$ wouldn't be maximal, a contradiction, which can only be resolved by assuming that

$$
\lim _{\tau \rightarrow a} \mathrm{e}^{f}=r_{0} .
$$

\section{Acknowledgment}

This work has been supported by the Deutsche Forschungsgemeinschaft.

\section{References}

[1] C. Gerhardt, Hypersurfaces of prescribed curvature in Lorentzian manifolds, Indiana Univ. Math. J. 49 (2000), 1125-1153; arXiv:math.DG/0409457.

[2] C. Gerhardt, The inverse mean curvature flow in ARW spaces - transition from big crunch to big bang, 2004, 39 pages, arXiv:math.DG/0403485.

[3] C. Gerhardt, The inverse mean curvature flow in Robertson-Walker spaces and its application to cosmology, (2004), 9 pages, gr-qc/0404112.

[4] C. Gerhardt, Transition from big crunch to big bang in brane cosmology, Adv. Theor. Math. Phys. 8 (2004), 319-343, 25 pages; gr-qc/0404061.

[5] P. Kraus, Dynamics of anti-de sitter domain walls, JHEP 12 (011) (1999); arXiv:hep-th/9910149.

[6] N. Seiberg, From big crunch to big bang - is it possible?, (2002), 12 pages, hep-th/0201039.

[7] N. Turok and P. J. Steinhardt, Beyond inflation: a cyclic universe scenario, (2004), 27 pages, hep-th/0403020. 
